\title{
Sums of Squares of Fibonacci Numbers with Prime Indices
}

\author{
A. Gnanam, B. Anitha \\ Department of Mathematics, Government Arts College, Tiruchirappalli, India \\ Email:gnaanam@yahoo.com, anithamaths2010@gmail.com
}

Received 18 September 2015; accepted 15 December 2015; published 18 December 2015

Copyright (C) 2015 by authors and Scientific Research Publishing Inc.

This work is licensed under the Creative Commons Attribution International License (CC BY). http://creativecommons.org/licenses/by/4.0/

(c) (i) Open Access

\section{Abstract \\ In this paper we present some identities for the sums of squares of Fibonacci and Lucas numbers with consecutive primes, using maximal prime gap $G(x) \sim \log ^{2} x$, as indices.}

\section{Keywords}

\section{Maximal Gap, Lucas Numbers, Fibonacci Numbers, Sums of Squares}

\section{Introduction}

The two most well-known linear homogeneous recurrence relations of order two with constant coefficients are those that define Fibonacci and Lucas numbers. $F_{n}$ denotes the $n^{\text {th }}$ Fibonacci number and $L_{n}$ denotes the $n^{\text {th }}$ Lucas number. The Lucas sequence is defined by $L_{n}=F_{n-1}+F_{n+1}$. The Fibonacci numbers are generated by the recursion $F_{n}=F_{n-1}+F_{n-2}$. The Lucas numbers also have the property that for $n>2, L_{n}=L_{n-1}+L_{n-2}$. Note that a Lucas number is always greater than its corresponding Fibonacci numbers except for $L_{1}$. Fibonacci and Lucas numbers are represented by the same recurrence relation. This is the reason that Fibonacci and Lucas numbers have so many common or very similar properties.

Hundreds of Fibonacci and Lucas identities involving both Fibonacci and Lucas numbers appeared in various journals [1]-[3] and books [4] [5] over the years. Our goal in this paper is to present some identities concerning sums of squares of Fibonacci and Lucas numbers with consecutive primes as indices. Regarding consecutiveness of primes we consider maximal gap between consecutive primes.

$G(x)$, the maximal gap between consecutive primes is defined by many approximations [6] [7]. Among those we observed that $G(x) \sim \log ^{2} x$ gives the value nearest to the actual value while calculating sums of squares of consecutive primes. Based upon this, here we have chosen $G(x) \sim \log ^{2} x$ and calculated sums of squares of Fibonacci and Lucas Primes. 


\section{Identities on Sums of Squares of Fibonacci and Lucas Numbers}

Here the following formulae are repeatedly used.

$$
\begin{gathered}
L_{n}=F_{n-1}+F_{n+1} \\
F_{n}=F_{n-1}+F_{n-2} \\
L_{n}=L_{n-1}+L_{n-2} \\
5 F_{2 n+1}=L_{n}^{2}+L_{n+1}^{2} \\
F_{2 n+1}=F_{n}^{2}+F_{n+1}^{2} \\
F_{n-1} F_{n+2}=F_{n+1}^{2}-F_{n}^{2} \\
F_{m} F_{m-1}=F_{m}^{2}-F_{m-1}^{2}+(-1)^{m} \\
F_{2 n-1}=F_{n}^{2}+F_{n-1}^{2}
\end{gathered}
$$

The difference between two consecutive primes $(p \geq 3)$ always being an even integer, the indices are taken as $p$ and $p+2 k, k \geq 1$ to calculate sums of squares of two Lucas numbers with indices as primes and its consecutive primes.

\subsection{Proposition}

$$
L_{p}^{2}+L_{p+2 k}^{2}=2 F_{2 p-1}+5 F_{2 p-3}-2 F_{2 p-5}-2 F_{2 p+4 k-5}+5 F_{2 p+4 k-3}+2 F_{2 p+4 k-1}-4
$$

where $k \geq 1$.

\section{Proof.}

Using the basic recurrence relation $L_{n}=L_{n-2}+L_{n-1}$, for an odd prime $p$ we have

$$
\begin{aligned}
L_{p}^{2}+L_{p+2 k}^{2}= & L_{p-2}^{2}+L_{p-1}^{2}+2 L_{p-2} L_{p-1}+L_{p+2 k-2}^{2}+L_{p+2 k-1}^{2}+2 L_{p+2 k-2} L_{p+2 k-1} \\
= & 5 F_{2 p-3}+5 F_{2 p+4 k-3}+2\left[\left(F_{p-3}+F_{p-1}\right)\left(F_{p-2}+F_{p}\right)+\left(F_{p+2 k-3}+F_{p+2 k-1}\right)\left(F_{p+2 k-2}+F_{p+2 k}\right)\right] \\
= & 5 F_{2 p-3}+5 F_{2 p+4 k-3}+2\left[F_{p-2} F_{p-3}+F_{p-1} F_{p-2}+F_{p-3} F_{p}+F_{p} F_{p-1}+F_{p+2 k-3} F_{p+2 k-2}\right. \\
& \left.+F_{p+2 k-3} F_{p+2 k}+F_{p+2 k-1} F_{p+2 k-2}+F_{p+2 k-1} F_{p+2 k}\right] \\
= & 5 F_{2 p-3}+5 F_{2 p+4 k-3}+2\left\{F_{p-2}^{2}-F_{p-3}^{2}+(-1)^{p-2}+F_{p-1}^{2}-F_{p-2}^{2}+(-1)^{p-1}+F_{p}^{2}-F_{p-1}^{2}\right. \\
& +(-1)^{p}+F_{p+2 k-2}^{2}-F_{p+2 k-3}^{2}+(-1)^{p+2 k-2}+F_{p+2 k-1}^{2}-F_{p+2 k-2}^{2}+(-1)^{p+2 k-1}+F_{p+2 k}^{2} \\
& \left.-F_{p+2 k-1}^{2}+(-1)^{p+2 k}+F_{p-1}^{2}-F_{p-2}^{2}+F_{p+2 k-1}^{2}-F_{p+2 k-2}^{2}\right\} \\
= & 5 F_{2 p-3}+5 F_{2 p+4 k-3}+2\left[\left(F_{p}^{2}+F_{p-1}^{2}\right)-\left(F_{p-3}^{2}+F_{p-2}^{2}\right)-\left(F_{p+2 k-2}^{2}+F_{p+2 k-3}^{2}\right)+\left(F_{p+2 k}^{2}+F_{p+2 k-1}^{2}\right)\right]-4 \\
& \quad L_{p}^{2}+L_{p+2 k}^{2}=2 F_{2 p-1}+5 F_{2 p-3}-2 F_{2 p-5}-2 F_{2 p+4 k-5}+5 F_{2 p+4 k-3}+2 F_{2 p+4 k-1}-4
\end{aligned}
$$

\section{Illustrations}

\begin{tabular}{cccc}
\hline$p$ & $k$ & $L_{p}^{2}+L_{p+2 k}^{2}$ & $2 F_{2 p-1}+5 F_{2 p-3}-2 F_{2 p-5}-2 F_{2 p+4 k-5}+5 F_{2 p+4 k-3}+2 F_{2 p+4 k-1}-4$ \\
\hline 3 & 2 & $4^{2}+29^{2}=857$ & $2(5)+5(2)-2(1)-2(34)+5(89)+2(233)-4=857$ \\
& & & $2(75025)+5(28657)-2(10946)-2(3524578)$ \\
13 & 3 & $521^{2}+9349^{2}=87675242$ & $+5(9227465)+2(24157817)-4=87675242$ \\
& & & \\
29 & 1 & $1149851^{2}+3010349^{2}=1.038435842 \times 10^{13}$ & $2(365435296162)+5(139583862445)-2(53316291173)-2(365435296162)$ \\
& & & $+5(956722026041)+2(2504730781961)-4=1.038435842 \times 10^{13}$
\end{tabular}


As the number of primes is infinite we try to use $\lfloor G(x)\rfloor$, the maximal gap between consecutive primes as indicies. Through there are many formulae available for $G(x)$, in [8]. It is observed that while calculating sums of squares of consecutive primes $\log ^{2} x$ gives the value nearest to the actual value. Hence $\lfloor G(x)\rfloor \sim \log ^{2} x$ has been used.

\subsection{Proposition}

Proof.

$$
L_{p}^{2}+L_{p+\lfloor G(x)\rfloor}^{2} \leq 2 F_{2 p-1}+5 F_{2 p-3}-2 F_{2 p-5}-2 F_{2 p+2\lfloor G(x)\rfloor-5}+5 F_{2 p+2\lfloor G(x)\rfloor-3}+2 F_{2 p+2\lfloor G(x)\rfloor-1}-4
$$

Again using the recurrence relation $L_{n}=L_{n-2}+L_{n-1}$ we have,

$$
\begin{aligned}
& L_{p}^{2}+L_{p+\lfloor G(x)\rfloor}^{2}=L_{p-2}^{2}+L_{p-1}^{2}+2 L_{p-2} L_{p-1}+L_{p+\lfloor G(x)\rfloor-2}^{2}+L_{p+\lfloor G(x)\rfloor-1}^{2}+2 L_{p+\lfloor G(x)\rfloor-2} L_{p+\lfloor G(x)\rfloor-1} \\
& =5 F_{2 p-3}+5 F_{2 p+2\lfloor G(x)\rfloor-3}+2\left[\left(F_{p-3}+F_{p-1}\right)\left(F_{p-2}+F_{p}\right)+\left(F_{p+\lfloor G(x)\rfloor-3}+F_{p+\lfloor G(x)\rfloor-1}\right)\left(F_{p+\lfloor G(x)\rfloor-2}+F_{p+\lfloor G(x)\rfloor}\right)\right] \\
& =5 F_{2 p-3}+5 F_{2 p+2[G(x)\rfloor-3}+2\left[F_{p-2} F_{p-3}+F_{p-1} F_{p-2}+F_{p-3} F_{p}+F_{p} F_{p-1}+F_{p+\lfloor G(x)\rfloor-3} F_{p+\lfloor G(x)\rfloor-2}\right. \\
& \left.+F_{p+\lfloor G(x)\rfloor-3} F_{p+\lfloor G(x)\rfloor}+F_{p+\lfloor G(x)\rfloor-1} F_{p+\lfloor G(x)\rfloor-2}+F_{p+\lfloor G(x)\rfloor-1} F_{p+\lfloor G(x)\rfloor}\right] \\
& =5 F_{2 p-3}+5 F_{2 p+2[G(x)\rfloor-3}+2\left\{F_{p-2}^{2}-F_{p-3}^{2}+(-1)^{p-2}+F_{p-1}^{2}-F_{p-2}^{2}+(-1)^{p-1}+F_{p}^{2}-F_{p-1}^{2}+(-1)^{p}\right. \\
& +F_{p+\lfloor G(x)\rfloor-2}^{2}-F_{p+\lfloor G(x)\rfloor-3}^{2}+(-1)^{p+\lfloor G(x)\rfloor-2}+F_{p+\lfloor G(x)\rfloor-1}^{2}-F_{p+\lfloor G(x)\rfloor-2}^{2}+(-1)^{p+\lfloor G(x)\rfloor-1}+F_{p+\lfloor G(x)\rfloor}^{2} \\
& \left.-F_{p+\lfloor G(x)\rfloor-1}^{2}+(-1)^{p+\lfloor G(x)\rfloor}+F_{p-1}^{2}-F_{p-2}^{2}+F_{p+\lfloor G(x)\rfloor-1}^{2}-F_{p+\lfloor G(x)\rfloor-2}^{2}\right\} \\
& =5 F_{2 p-3}+5 F_{2 p+2[G(x)\rfloor-3}+2\left[\left(F_{p}^{2}+F_{p-1}^{2}\right)-\left(F_{p-3}^{2}+F_{p-2}^{2}\right)+\left(F_{p+\lfloor G(x)\rfloor}^{2}+F_{p+\lfloor G(x)\rfloor-1}^{2}\right)-\left(F_{p+\lfloor G(x)\rfloor-2}^{2}+F_{p+\lfloor G(x)\rfloor-3}^{2}\right)\right]-4 \\
& \quad L_{p}^{2}+L_{p+\lfloor G(x)\rfloor}^{2} \leq 2 F_{2 p-1}+5 F_{2 p-3}-2 F_{2 p-5}-2 F_{2 p+2\lfloor G(x)\rfloor-5}+5 F_{2 p+2[G(x)\rfloor-3}+2 F_{2 p+2\lfloor G(x)\rfloor-1}-4
\end{aligned}
$$

Now using Binet's formula, sums of squares of two Fibonacci numbers with consecutive primes as indicies has been expressed in terms of Lucas numbers.

\subsection{Proposition}

Proof.

$$
F_{p}^{2}+F_{p+2 k}^{2}=\frac{1}{5}\left[L_{2 p}+L_{2(p+2 k)}+4\right]
$$

In general

$$
\begin{gathered}
F_{p}^{2}=\left[\frac{\alpha^{p}-\beta^{p}}{\sqrt{5}}\right]^{2}=\frac{1}{5}\left[\alpha^{2 p}+\beta^{2 p}-2(-1)^{p}\right] \\
F_{p}^{2}=\frac{1}{5}\left[L_{2 p}-(-1)^{p}\right]
\end{gathered}
$$

$$
\begin{gathered}
F_{p+2 k}^{2}=\frac{1}{5}\left[\alpha^{2(p+2 k)}+\beta^{2(p+2 k)}-2(-1)^{p+2 k}\right] \\
F_{p+2 k}^{2}=\frac{1}{5}\left[L_{2(p+2 k)}-2(-1)^{(p+2 k)}\right] \\
F_{p}^{2}+F_{p+2 k}^{2}=\frac{1}{5}\left[L_{2 p}-2(-1)^{p}\right]+\frac{1}{5}\left[L_{2(p+2 k)}-2(-1)^{p+2 k}\right] \\
=\frac{1}{5}\left[L_{2 p}+L_{2(p+2 k)}-2\left[(-1)^{p}+(-1)^{p+2 k}\right]\right]
\end{gathered}
$$




$$
F_{p}^{2}+F_{p+2 k}^{2}=\frac{1}{5}\left[L_{2 p}+L_{2(p+2 k)}+4\right]
$$

\section{Illustrations}

\begin{tabular}{cccc}
\hline$p$ & $k$ & $F_{p}^{2}+F_{p+2 k}^{2}$ & $\frac{1}{5}\left[L_{2 p}+L_{2(p+2 k)}+4\right]$ \\
\hline 5 & 6 & $5^{2}+1597^{2}=2550434$ & $\frac{1}{5}(123+12752043+4)=2550434$ \\
11 & 4 & $89^{2}+4181^{2}=17488682$ & $\frac{1}{5}(39603+87403803+4)=17488682$ \\
23 & 1 & $28657^{2}+75025^{2}=6449974274$ & $\frac{1}{5}(4106118243+28143753123+4)=6449974274$ \\
\hline
\end{tabular}

As discussed in proposition 2.2, $G(x)$ has been taken for this proposition also.

\subsection{Proposition}

$$
F_{p}^{2}+F_{p+\lfloor G(x)\rfloor}^{2} \leq \frac{1}{5}\left[L_{2 p}+L_{2(p+\lfloor G(x)\rfloor)}+4\right]
$$

Proof.

$$
\begin{aligned}
F_{p}^{2}+F_{p+\lfloor G(x)\rfloor}^{2} & =\frac{1}{5}\left[L_{2 p}-2(-1)^{p}\right]+\frac{1}{5}\left[L_{2(p+\lfloor G(x)\rfloor)}-2(-1)^{p+\lfloor G(x)\rfloor}\right] \\
& =\frac{1}{5}\left[L_{2 p}+L_{2(p+\lfloor G(x)\rfloor)}-2\left[(-1)^{p}+(-1)^{p+\lfloor G(x)\rfloor]]}\right.\right. \\
F_{p}^{2} & +F_{p+\lfloor G(x)\rfloor}^{2} \leq \frac{1}{5}\left[L_{2 p}+L_{2(p+\lfloor G(x)\rfloor)}+4\right]
\end{aligned}
$$

Finally, sums of squares of a Fibonacci and Lucas number is found.

\subsection{Proposition}

$$
L_{n}^{2}+F_{n}^{2}=\frac{1}{5}\left[6 L_{2 n}+2^{3}(-1)^{n}\right]
$$

\section{Proof.}

Using Binet's formula, we have

$$
\begin{gathered}
L_{n}^{2}=\left(\alpha^{n}+\beta^{n}\right)^{2} \\
L_{n}^{2}=\alpha^{2 n}+\beta^{2 n}+2(\alpha \beta)^{n} \\
F_{n}^{2}=\frac{1}{5}\left(\alpha^{2 n}+\beta^{2 n}-2(\alpha \beta)^{n}\right) \\
L_{n}^{2}+F_{n}^{2}=\alpha^{2 n}+\beta^{2 n}+2(\alpha \beta)^{n}+\frac{1}{5}\left(\alpha^{2 n}+\beta^{2 n}-2(\alpha \beta)^{n}\right) \\
=\alpha^{2 n}+\beta^{2 n}+\frac{1}{5}\left(\alpha^{2 n}+\beta^{2 n}\right)+2(-1)^{n}-\frac{2}{5}(-1)^{n} \\
L_{n}^{2}+F_{n}^{2}=L_{2 n}+\frac{1}{5} L_{2 n}+\frac{2^{3}}{5}(-1)^{n} \\
5\left(L_{n}^{2}+F_{n}^{2}\right)=6 L_{2 n}+2^{3}(-1)^{n}
\end{gathered}
$$




\section{References}

[1] Azarian, M.K. (2012) Identities Involving Lucas or Fibonacci Numbers as Binomial Sums. International Journal of Contemporary Mathematical Sciences, 7, 2221-2227.

[2] Rathore, G.P.S., Singh, B. and Jhala, D. (2014) Some Identities Involving Common Factors of k-Fibonacci and kLucas Numbers. American Journal of Mathematical Analysis, 3, 33-35.

[3] Ramfrez, J.L. (2013) Incomplete Generalized Fibonacci and Lucas Polynomial. Hacettepe Journal of Mathematics and Statistics, 44, 363-373.

[4] Hoggatt Jr., V.E. (1979) Fibonacci and Lucas Numbers. The Fibonaci Association, University of Santaclara, CA.

[5] Koshy, T. (2001) Fibonacci and Lucas Numbers with Applications. A Wiley-Interscience Publication, John Wiley \& Sons, Inc.

[6] Wolf, M. (1997) First Occurrence of a Given Gap between Consecutive Primes.

[7] Cadwell, J.H. (1971) Large Intervals between Consecutive Primes. Mathematics of Computation, 25, 909-913. http://dx.doi.org/10.1090/S0025-5718-1971-0299567-6

[8] Falcon, S. (2012) On the k-Lucas Numbers of Arithmetic Indexes. Applied Mathematics, 3, 1202-1206. http://dx.doi.org/10.4236/am.2012.310175

\section{Notation}

$\lfloor x\rfloor$ (The Floor of $x$ ) - The Greatest Integer $\leq x$ 